\title{
Pharmacotherapy of Retinal Disease with Visual Cycle Modulators
}

Rehan M. Hussain, M.D. ${ }^{1}$, Ninel Z. Gregori, M.D. ${ }^{1}$, Thomas A. Ciulla, M.D., M.B.A. ${ }^{2,3}$, and Byron L. Lam, M.D. ${ }^{1}$

1. Bascom Palmer Eye Institute, University of Miami Miller School of Medicine, 900 NW $17^{\text {th }}$ St, Miami, FL 33136

2. Retina Service, Midwest Eye Institute, 200 W. $103^{\text {rd }}$ St, Indianapolis, IN 46290

3. Indiana University School of Medicine, $340 \mathrm{~W} 10^{\text {th }} \mathrm{St}$, Indianapolis, IN 46202

Corresponding Author:

Rehan M. Hussain, MD

Bascom Palmer Eye Institute

University of Miami Miller School of Medicine

$900 \mathrm{NW} 17^{\text {th }}$ St, Miami FL 33136

rhussain27@med.miami.edu

Phone: $708-288-5540$

Fax: 305-326-6580

Disclosures:

Dr. Ciulla had an employment relationship with, and equity ownership in, Ophthotech corporation during manuscript conception and initial preparation. He subsequently had an employment relationship with, and equity ownership in, Spark Therapeutics. This work was undertaken in his role as Volunteer Clinical Professor at Indiana University School of Medicine, and does not reflect any views or opinions of these corporations or management. Dr. Lam serves as a consultant for Spark Therapeutics and receives study funding from Sanofi, StarGen, and Alkeus. Drs. Hussain and Gregori have no financial disclosures.

This is the author's manuscript of the article published in final edited form as:

Hussain, R. M., Gregori, N. Z., Ciulla, T. A., \& Lam, B. L. (2018). Pharmacotherapy of retinal disease with visual cycle modulators. Expert Opinion on Pharmacotherapy, 19(5), 471-481. https://doi.org/10.1080/14656566.2018.1448060 


\begin{abstract}
$\underline{\text { Abstract }}$
Introduction: Pharmacotherapy with visual cycle modulators (VCMs) is under investigation for multiple diseases, including retinitis pigmentosa (RP), Leber congenital amaurosis (LCA), Stargardt macular dystrophy (SMD) and nonexudative age-related macular degeneration (AMD), all blinding diseases that lack effective treatment options.
\end{abstract}

Areas Covered: Oral retinoids, including 9-cis-retinyl-acetate (Zuretinol) and 9-cis- $\beta$ carotene, restore 11-cis-retinal levels in RP and LCA caused by LRAT and RPE65 gene mutations, and may improve visual acuity and visual fields. Therapies for SMD aim to decrease accumulation of toxic Vitamin A dimers and lipofuscin in the retina and retinal pigment epithelium (RPE) include C20-D3-vitamin A (ALK-001), isotretinoin, VM200 (aldehyde trap), emixustat, and A1120. Mouse models of SMD show promising data for these treatments, though proof of efficacy in humans is currently lacking. Fenretinide and emixustat are investigational VCMs for dry AMD, though neither has been shown to reduce geographic atrophy or improve vision in human trials. A1120 prevents retinol transport into the RPE and may spare the side effects typically seen in VCMs (nyctalopia and chromatopsia) according to mouse studies.

Expert Opinion: Oral VCMs may be feasible treatment options for degenerative retinal diseases based on pre-clinical studies. Further trials are warranted to assess their efficacy and safety in humans. 
Keywords: 9-cis-retinyl-acetate, C20-D3-vitamin A, emixustat, fenretinide, geographic atrophy, Leber's Congenital Amaurosis, Retinitis Pigmentosa, Stargardt macular dystrophy, visual cycle

\section{The Visual Cycle}

The visual cycle refers to an enzymatic process that takes place in the outer retina photoreceptors and retinal pigment epithelium (RPE), in which light is converted into an electrical signal that is ultimately conveyed to the brain. As understanding of the visual cycle progresses, the ability to manipulate the visual cycle dysfunction to treat retinal disease is becoming a reality.

The rods are single photon receptors that allow visual perception in low illumination, while the cones are less sensitive but can distinguish various wavelengths of light, allowing for color vision. Both rods and cones use 11-cis-retinal, which binds to opsins to then form visual pigments such as rhodopsin or cone opsins [1]. When light strikes rhodopsin (composed of the protein opsin bound to the chromophore 11-cisretinal, a vitamin A derivative) in the rod outer segments, 11-cis-retinal is converted to its all-trans-retinal isomer. This, in turn, activates the opsin and initiates a signal transduction cascade, closing a cyclic GMP-gated cation channel, and hyperpolarizing the photoreceptor cell. In the visual cycle, the all-trans-retinal must be converted back to 11cis-retinal, via a series of steps catalyzed by enzymes, including retinol dehydrogenases $(\mathrm{RDH})$, which catalyze reduction and oxidation reactions in the photoreceptor, as well as lecithin retinol acyltransferase (LRAT) and retinoid isomerohydrolase (a 65 kilodalton 
protein, encoded by the RPE65 gene), both of which are located in the RPE [2]. The visual cycle is diagrammed in Figure 1, which also shows the role of select therapeutics that influence the visual cycle.

Within the visual cycle, the ATP binding cassette subfamily A member 4 (ABCA4) is localized to the outer segments of photoreceptors and acts as a membrane transporter for the recycling of chromophore during the visual cycle. Specifically, within the visual cycle, ABCA4 binds with high affinity and shuttles $N$ retinylidene-phosphatidylethanolamine trapped inside the photoreceptor disc across the membrane surface, where it is ultimately further processed by the adjacent retinal pigment epithelial cells.

The visual cycle plays a key role in several retinal disorders. For example, dysfunction of enzymes in the visual cycle leads to several inherited retinal diseases (IRDs) such as retinitis pigmentosa (RP), Leber's congenital amaurosis (LCA), and Stargardt macular dystrophy (SMD), due to the inability to either produce an adequate supply of 11-cis-retinal or an inability to remove the accumulation of various retinoid products. The visual cycle has become the focus of therapeutic strategies as several compounds have the potential to address defects in this cycle to treat rare IRDs. Several clinical trials have utilized these visual cycle modulators in an attempt to slow the progression of SMD and age-related macular degeneration (AMD), the leading cause of irreversible blindness in the industrialized world. The drugs discussed in this review are summarized in Table 1.

\section{Dysfunction Within the Visual Cycle and Therapies to Address Dysfunction}




\subsection{LRAT and RPE65 Dysfunction: Inherited Retinal Disease}

Retinitis pigmentosa (RP) is the most common IRD, with a prevalence of approximately 1 in 3500 [3]. The term RP describes a group of genetically-heterogeneous progressive rod-cone retinal degenerations. Patients typically develop night blindness in the early phase of the disease, followed by loss of mid-peripheral visual field with progressive visual loss towards central vision [4]. Figure 2 shows a montage fundus photo of a patient with RP, which demonstrates the classic triad of optic disc pallor, retinal vessel attenuation, and "bone-spicule" pigmentary changes in the retinal periphery.

Leber congenital amaurosis (LCA) is a group of congenital or early onset forms of RP, with an autosomal recessive inheritance pattern, that occurs in approximately one in 81,000 births and inevitably leads to severe early visual impairment [5]. Patients with LCA typically have poor central and peripheral vision, early-onset nystagmus and severely diminished electroretinogram (ERG) responses. Figure 3 shows a montage fundus photo of a patient with LCA, which demonstrates retinal vessel attenuation and pigmentary changes similar to those seen in RP.

Genes mutated in IRDs encode proteins that are involved in multiple mechanisms and pathways, including the phototransduction cascade (which includes the visual cycle described above), maintenance of photoreceptor structure, gene transcription and ciliary function. Mutations in more than 200 genes have been identified in IRDs. These genes account for only about $60 \%$ of all patients; whereas the remainder have defects in unidentified genes [3]. Mutations in LRAT and RPE65 are among those known to cause 
RP and LCA $[6,7]$. As noted above, RPE65 and LRAT are responsible for production and recycling of 11-cis-retinal and rhodopsin. Deficiencies of 11-cis retinal and rhodopsin cause severe rod and cone dysfunction leading to retinal degeneration and several visual loss [8]

\subsubsection{Bypass of LRAT and RPE65 Visual Cycle Defects: 9-cis-retinyl-acetate}

\section{(QLT091001, Zuretinol)}

In models of LRAT and RPE65-deficient mice, treatment with the chromophore pro-drug, 9-cis-retinyl-acetate, prevented loss of cone photoreceptors (examined histologically) and partially preserved the ERG b-wave compared to mice in the control group [9]. 9-cis-retinyl-acetate combines with opsin to form isorhodopsin, which is also capable of starting the phototransduction cascade when activated by light [10]. Essentially, 9-cis-retinal administration can bypass defects in the visual cycle and regenerate visual pigment as isorhodopsin, thereby restoring visual function and ameliorating the progression of retinal degeneration in LRAT and RPE65-deficient animals. High doses of prolonged 9-cis-retinyl-acetate administration are well tolerated by the retinas of the enzyme-deficient mice, as determined by preservation of retinal thickness and morphology on spectral-domain optical coherence tomography (SD-OCT); no significant increases in fundus autofluorescence were detected at 6 months [11].

Batten et al demonstrated visual functions could be rescued in LRAT-deficient mice, as measured by recovery of levels of visual chromophore and pigment and improved ERG responses. Successful restoration of retinal signaling, as measured by pupillary responses, was also achieved. Treatment with orally administered pro-drugs 9- 
cis-retinyl acetate and 9-cis-retinyl succinate (which chemically circumvent the LRATcatalyzed step in chromophore regeneration) caused enduring renewal of retinal function in LRAT-deficient mice and amplified ERG response from roughly $5 \%$ of wild-type levels in $\mathrm{LRAT}^{-/-}$mice to approximately $50 \%$ of wild-type levels in treated $\mathrm{LRAT}^{-/}$mice. In the same study, the authors also showed that gene therapy with intraocular injection of recombinant adeno-associated virus (AAV) carrying the LRAT gene successfully restored ERG responses; oral and gene therapy techniques were complementary when combined. While full restoration of the ERG could not be obtained in $\mathrm{LRAT}^{-/-}$mice in the tested experimental conditions, the finding of nearly complete restoration of single cell responses suggests that remodeling of the neuronal retina in $\mathrm{LRAT}^{-/-}$may limit functional rescue [12].

In a human phase $1 \mathrm{~b}$ trial, patients with LCA or early onset RP caused by mutations in RPE65 (78\%) or LRAT (22\%) were given once daily oral 9-cis-retinylacetate (also known as QLT091001 or Zuretinol acetate - developed by Novelion Therapeutics) $40 \mathrm{mg} / \mathrm{m}^{2} /$ day for 7 days and many were found to have improvement in visual field and best-corrected visual acuity (BCVA) [13]. Eight of 18 (44\%) patients showed a $\geq 20 \%$ increase and 4 of $18(22 \%)$ showed a $\geq 40 \%$ increase in functional retinal area as measured by Goldmann visual fields (GVF). $12(67 \%)$ and $5(28 \%)$ of 18 patients showed a $\geq 5$ and $\geq 10$ ETDRS letter score increase of visual acuity, respectively, in one or both eyes at two or more visits within 2 months of treatment. The specific genotypes (LRAT or RPE65) did not appear to affect how patients responded to the drug [13]. There were no detectable improvements observed on standard electroretinography (ERG), similar to what has been previously observed in gene therapy trials for LCA [14-16]. 
In the same phase $1 \mathrm{~b}$ trial, which included 14 patients with LCA caused by LRAT and RPE65 mutations, treatment with a 7-day course of 9-cis-retinyl-aceteate (QLT091001, 10-40 mg/m²) was followed by an improvement in GVF areas (mean increase in retinal area of $28-683 \%$ ) in 10 patients (71\%) for a median duration of 163 days [16]. Patients with small to medium baseline GVF had greater improvement than those with a larger baseline GVF. Six patients (43\%) had an improvement in visual acuity (mean increase of 2-30 letters) along with subjective reporting of improvement in activities of daily living. The median duration of the visual acuity response was 315 days (interquartile range: 111-534 days). After 2 years, three (21\%) patients had a sustained GVF response and four (30\%) had a sustained visual acuity response. ERG function did not improve in this trial, possibly due to the level of retinal degeneration already present at initiation of therapy. Four patients had functional MRI scans, which all correlated with visual response - there was increased cortical activation in one patient with increased GVF area, and there was no increased cortical activation in three patients that did not show increase in GVF area. No serious adverse events were noted, though 6 patients (43\%) developed moderate-severe headache that resolved within 1-2 days of the last treatment of QLT091001, 7 patients developed moderate-severe photophobia that corresponded with visual improvement, and 8 patients (57\%) had a transient increase in serum triglyceride levels [17].

Measuring the outer segment length of the photoreceptor layer with SD-OCT was highly predictive of treatment responses, with responders having a significantly larger baseline outer segment thickness $(11.7 \pm 4.8 \mu \mathrm{m}$, mean $\pm 95 \% \mathrm{CI})$ than non-responders $(3.5 \pm 1.2 \mu \mathrm{m})$. This structure-function relationship suggests that treatment with 
QLT091001 is more likely to be efficacious if there is sufficient photoreceptor integrity [13]. According to the Novelion website, 9-cis-retinyl-acetate (Zuretinol) has Orphan Drug designation from both the U.S. Food and Drug Administration (FDA) and European Medicines Agency, along with FDA Fast Track designation. [18].

\subsubsection{Bypass of LRAT and RPE65 Visual Cycle Defects: 9-cis $\beta$-carotene}

Another randomized placebo-controlled crossover trial (NCT01256697) examined the effect of 9-cis- $\beta$-carotene-rich powder in patients with RP of various genotypes [19]. Oral 9-cis $\beta$-carotene can access the retina, where it can be converted to 9-cis-retinal and combine with opsin to form isorhodopsin [20]. The retinoid 9-cis-retinal, which has a light absorption spectrum similar to that of 11-cis-retinal, can replace the latter when its availability is limited owing to a retinoid cycle defect. 9-cis-retinal also induces an increase in the endogenous production of 11-cis-retinal, as observed in mice with a different inborn error in the retinoid cycle [10]. Furthermore, the 9-cis $\beta$-carotene or its metabolites may reduce inflammation and serve as an antioxidant [21].

29 participants completed the trial, in which they were treated daily for 90 days with 4 capsules each containing $300 \mathrm{mg}$ of alga Dunaliella bardawil (a 9-cis- $\beta$-carotenerich powder, for a total approximately $20 \mathrm{mg}$ of $\beta$-carotene) or placebo. The over-thecounter Dunaliella capsules are approved by the US FDA and have been used for more than 20 years with no known significant adverse effects. Following a 90-day washout period, the groups crossed over and received treatment with the other capsule for 90 days. 
This crossover design allows for each patient to receive both treatments, thus allowing each participant to serve as their own control for comparison. The primary outcome of the study was the dark-adapted ERG maximal b-wave response amplitude, representing rod and cone function objectively. The 4 secondary outcomes were light-adapted ERG bwave response amplitudes (objective cone function), dark adapted chromatic visual field area (subjective rod or cone function), conventional light-adapted visual field area (subjective cone and rod function), and BCVA. The measurements were obtained at baseline, at the end of the first 90 day treatment period, at the end of the 90 day washout period, and at the end of the second 90 day treatment period [19].

In $10 / 29$ patients $(34.5 \%, 5$ patients in each group), the maximal b-wave response increased by more than $10 \mu \mathrm{V}$ (range $11-42 \mu \mathrm{V}$ ) in both eyes. Relative to initial baseline, treatment with Dunaliella yielded a significant increase in maximal dark-adapted ERG bwave amplitude $(+8.4 \mu \mathrm{V})$, whereas placebo treatment resulted in a decrease $-5.9 \mu \mathrm{V}$ $(p=0.001)$. The percentage change in light-adapted $b$-wave response was $+17.8 \%$ for 9 cis $\beta$-carotene vs $-3.0 \%$ for placebo $(\mathrm{P}=.01)$. Thus, both objective measures (rod and cone photoresponse functions) showed significant improvements after 90 days of Dunaliella treatment. No significant differences were found between the groups for visual field and BCVA. No adverse effects were observed. Based on these results, the authors concluded that 9-cis- $\beta$-carotene may serve as a new therapeutic approach for some patients with RP, possibly as an adjuvant to gene therapy. Further trials with larger groups of patients and a larger washout period are planned for the future [19]. 


\subsubsection{RPE65 Gene Therapy}

Another area of research focus for LCA and RP is gene therapy to correct RPE65 mutations. Although a comprehensive review of genetic therapy options is beyond the scope of this review, it is worth noting that Spark Therapeutics completed a phase 3 trial of its adeno-associated viral vector containing RPE65 complementary DNA (called SPKRPE65), which is delivered via subretinal injection. To fully assess the functional benefits of RPE65 gene therapy in IRD patients, a proprietary multiluminance mobility test (MLMT) was developed, with feedback from the FDA in order to more functionally assess VA, visual fields, light sensitivity, and mobility [22]. In this test, patients progress through a mobility test with 12 standardized templates, under multiple standardized lighting conditions, to determine the lowest illumination under which they can successfully navigate the course in under 3 minutes. The seven levels of illumination reproduce the lighting conditions encountered in daily life, from 1 lux, simulating a moonless night, to 400 lux, simulating an office setting. A change in score, based on illumination level, functions as the endpoint and has been validated.

Spark initiated the Phase III trial in November 2012 enrolling 31 patients with a mean age of 15 years; the patients were randomized 2:1 into control or receiving $1.5 \mathrm{x}$ $10^{11} \mathrm{vg}$ of SPK-RPE65 in both eyes within 18 days. After the 12-month time point, the control subjects were eligible to cross over into the treatment group. In October 2015, Spark announced positive top-line results. There were no serious adverse events (SAEs) or significant immune responses related to SPK-RPE65. The trial met its primary endpoint of change at one year in the bilateral MLMT from baseline $(p=0.001)$, with an average improvement 1.8 light levels in the treatment group versus 0.2 light levels in the 
control group. Of the 20 subjects randomized to initial treatment, $13(65 \%)$ were able to pass the MLMT at the lowest light level (1 lux, demonstrating maximal possible improvement) at one year versus none in the control group. Secondary endpoints successfully met included full-field light sensitivity threshold testing (full-field scotopic threshold) with 100-fold improvement in light sensitivity in treated subjects $(\mathrm{p}<0.001)$ and MLMT change score for the first injected eye $(\mathrm{p}=0.001)$. There was a mean improvement in VA of 9 ETDRS letters, although this did not reach statistical significance $(p=0.17)$ [23]. In May 2017, Spark announced that it had completed its rolling Biologics License Application (BLA) submission to the FDA for SPK-RPE65, Voretigene Neparvovec [24].

Other companies that are sponsoring gene therapy trials for LCA include Applied Genetics Technology Corporation (AGTC) [25] and MeiraGTx. MeiraGTx has initiated a phase 1 clinical trial of AAV-OPTRPE65 [26].

\subsection{ABCA4 Dysfunction: Stargardt Macular Dystrophy}

Autosomal recessive Stargardt macular dystrophy (SMD) is a dystrophy resulting from mutations in the ABCA4 (ABCR) gene. It is the most common form of inherited juvenile macular degeneration, affecting roughly in 1 in 10,000 people. Mutations in ABCA4 also result in RP and cone-rod dystrophy. The age of onset of juvenile and early adult SMD is usually 8-25 years with some cases occurring in older adults (late-adult onset SMD) $[27,28]$. A hallmark of the disease is premature accumulation of lipofuscin (the "wear and tear cellular debris") in the retinal pigment epithelium (RPE) of the eye, causing a pattern of yellowish flecks that extend outward from the macula (see Figure 4). 
The brown-yellow, autofluorescent, electron-dense material is also found in cells of the liver, kidney, heart muscle, adrenals, nerve, and ganglion and is considered one of the most consistent morphologic features of aging, with a rate of accumulation inversely related to longevity $[29,30]$. The RPE is critical for the neurosensory retina homeostasis; it acts as a transport exchange system with blood capillaries and is critical for regeneration and phagocytosis of photoreceptor outer segments. It is hypothesized that when RPE lipofuscin reaches a critical level, it contributes to a decline in cell function, resulting in the degeneration of the macular region with subsequent loss of central vision $[31,32]$.

The faulty gene in SMD, ABCA4, encodes for an outer segment rim protein (RmP). The function of RmP is to transport the all-trans -retinaldehydephosphatidylethanolamine (retinaldehyde-PE) Schiff base from the luminal side of the disk membrane to the cytosolic face, where retinaldehyde can then be transformed back to retinol [33]. Without its functional transporter, the retinaldehyde-PE conjugates may react to form vitamin A dimers (including A2E and ATR-dimer), which then accumulate in the RPE after phagocytosis of the photoreceptors outer segments. Vitamin A dimers are toxic to cultured RPE cells and are thought to play a significant role in lipofuscin formation and subsequent retinal degeneration [34, 35]. A2E sensitizes RPE cells to light-induced apoptosis [36] and has an inhibitory effect on phospholipid turnover in RPE phagolysosomes [37].

\subsubsection{Decrease Toxic Byproducts of ABCA4 Dysfunction: ALK-001 (C20-D3-}




\section{vitamin A)}

Kaufman and colleagues have shown that the rate determining step in vitamin A dimerization is the cleavage of a C20 carbon-hydrogen bond of the retinaldehyde-PE Schiff base [38]. Replacing the C20 hydrogen atoms of vitamin A with deuterium atoms (i.e. C20-D3 -vitamin A) makes this bond harder to cleave and impedes vitamin A dimerization. Several studies have sought to determine whether slowing the intrinsic reactivity of vitamin A to dimerize could slow lipofuscin formation in the RPE and delay changes associated with human SMD. Ma and colleagues raised $\mathrm{ABCA} 4^{-/-}$mutant albino mice (the mouse model of human SMD) on diets containing either C20-D3 -vitamin A (the treated group) or vitamin A at its natural isotopic abundance (the control group) and measured the concentration of vitamin A dimers, lipofuscin and other biological markers indicative of ocular health in both groups. Treated mice exhibited an $80 \%$ reduction in A2E, a 95\% reduction in ATR dimer and a 70\% decrease in fundus autofluorescence at three months of age. After six months, the treated group showed fewer lipofuscin granules as visualized qualitatively by electron microscopy, and at 12 months they showed improved eye function as measured by ERG. These results suggest that pathological phenotypes that arise from defects in the ABCA4 gene may result from the dimerization of vitamin A and may be improved by hindering the ability of vitamin A to dimerize [39].

Similar results were found in another mouse model of SMD, in which Vitamin A dimerization contributed to over $50 \%$ of lipofuscin accumulation and caused transcriptional dysregulation of several complement genes associated with inflammation [40]. Replacing Vitamin A with C20-D3-vitamin A impeded dimerization of Vitamin A 
(by approximately fivefold for A2E), and additionally normalized the aberrant transcription of complement genes without impairing retinal function. Phenotypic rescue by C20-D3-vitamin A was also observed noninvasively by quantitative autofluorescence in as little as 3 months after the initiation of treatment, whereas upon interruption of treatment, the age-related increase in autofluorescence resumed. These results further indicate that administration of C20-D3 -vitamin A may be a feasible therapeutic approach to slow the progression of associated retinal disease caused by Vitamin A dimerization. During these mice studies, no side effects were noted, and the animals were administered the drug for 12 months.

The promising results of the aforementioned pre-clinical studies have paved the way for the oral once-daily C20-D3-vitamin A molecule, ALK-001 (Alkeus Pharmaceuticals, Boston, MA), to begin human clinical trials in SMD. A Phase 1 trial (NCT02230228) to assess the safety and pharmacokinetics in healthy volunteers has been completed [41]. The phase 2 TEASE study (NCT02402660) is ongoing [42].

\subsubsection{Decrease Toxic Byproducts of ABCA4 Dysfunction: VM200}

Vision Medicine's VM200 molecule for SMD is currently in pre-clinical trials. This oral drug sequesters the toxic compound, all-trans retinal, to prevent retinal cell death [43]. Specifically, VM200 is a primary amine that reacts with the aldehyde group of all-trans retinal to form an inactive Schiff base, thus making it unable to form A2E. VM200 was shown to preserve retinal structure in $\mathrm{ABCA} 4^{-/-} \mathrm{Rdh} 8^{-/-}$mice, as measured by SD-OCT. According to unpublished data from Case Western Reserve University, VM200 has also demonstrated ability to preserve retinal function, as mice treated with it 
were noted to have increased concentration of 11-cis retinal (a biomarker of intact photoreceptors) compared to controls [44]. No significant toxicities were noted in 2-week and 13-week long studies. The molecule of VM200 is an enantiomer of pregabalin, which is used to treat neuropathic pain, though its affinity for the pregabalin target is 10 -fold less than that of pregabalin. VM200 is also indicated for other inborn errors of aldehyde metabolism including Sjogren-Larsson Syndrome, Best Disease, and Succinic semialdehyde-dehydrogenase deficiency. Pre-clinical studies are continuing [44].

\subsubsection{ABCA4 Gene Therapy}

Gene therapy is also being explored to treat SMD. The ABCA4 gene $(6.8 \mathrm{~kb})$ far exceeds the 4.5-5.0 kb capacity of the AAV vector and has required utilization of an equine infectious anemia lentivirus (EIAV) for gene transfer [45]. Subretinal injection of EIAV-ABCA4 was found to be effective in a knock out mouse model and the StarGen (Sanofi) gene replacement lentivector carrying the ABCA4 gene is currently in Phase 1/2 trials [46] [47].

3 Therapeutic Inhibition of the Visual Cycle

3.1 11-cis-retinol dehydrogenase

\subsubsection{Isotretinoin}

Isotretinoin (Accutane) is a drug that is used to treat acne, but has also been shown to inhibit lipofuscin formation in a mouse model [48]. It works by inhibiting 11cis-retinol dehydrogenase in the visual cycle, thus slowing the synthesis of 11-cisretinaldehyde and regeneration of rhodopsin. This explains the side effect of decreased 
night vision in patients who use isotretinoin for acne [49], though isotretinoin has not been shown to induce photoreceptor degeneration, and actually protects against lightinduced damage [50]. Light activation of rhodopsin results in its release of all-transretinaldehyde, which constitutes the first reactant in toxic $\mathrm{A} 2 \mathrm{E}$ biosynthesis. $\mathrm{ABCA} 4^{-/-}$ mice that were injected with isotretinoin had decreased production of A2E, along with less formation of lipofuscin granules in the retina compared to controls, as viewed by electron microscopy. Additionally, wildtype mice treated with isotretinoin for 2 months had a $40 \%$ reduction of $\mathrm{A} 2 \mathrm{E}$ formation in the RPE compared to controls. On ERG, both wild-type and $\mathrm{ABCA} 4^{-/-}$mice showed smaller delays in dark adaptation after isotretinoin administration with bright compared with dim probe flashes. These results suggest that isotretinoin reduced rhodopsin levels in both wild-type and $\mathrm{ABCA} 4^{-/-}$retinas. The authors propose that isotretinoin may delay visual loss in SMD and other retinal diseases linked to lipofuscin accumulation.

\subsection{RPE65}

\subsubsection{Emixustat.}

Emixustat (ACU-4429, developed by Acucela Inc) is a small non-retinoid derivative of retinylamine that inhibits RPE65, thus reducing the conversion of all-transretinyl ester to 11-cis-retinol and preventing accumulation of A2E. Phase 1 studies showed that the drug was well-tolerated up to $75 \mathrm{mg}$ with expected dose dependent suppression of scotopic ERG in healthy subjects [51]. It was initially developed to slow the progression of geographic atrophy in age-related macular degeneration (AMD), but it also being tested for SMD. 
AMD is the leading cause of irreversible legal blindness in people $\geq 65$ years of age in the Western world, affecting over 1.7 million people in the United States and expected to reach almost 3 million by 2020 [52]. AMD is classified into one of two general subgroups: the non-neovascular (non-exudative or dry) form of the disease and the neovascular (exudative or wet) form of the disease. The non-neovascular form of AMD accounts for approximately $90 \%$ of all AMD cases, and is often characterized by a slow degeneration of the macula, resulting in atrophy of the central macula with gradual vision loss over a period of years. In contrast, neovascular AMD (nAMD), although less prevalent, commonly causes sudden and often substantial loss of central vision, accounting for most cases of severe vision loss in this disease. This type of AMD is characterized by the growth of pathologic choroidal neovascularization (CNV) beneath the macula, which causes exudation of blood and/or fluid into the macula. Ultimately, neovascular tissue and associated exudates develop into a destructive macular scar, leading to central blindness.

The advanced stage of dry AMD, geographic atrophy (GA), is characterized by loss of retinal photoreceptors, RPE, and choriocapillaris, and is responsible for approximately $20 \%$ of all cases of legal blindness in North America. Currently, the major unmet need in AMD is there is no available treatment for GA. Figure 5 shows a color fundus photo of a patient with GA, which demonstrates a patch of atrophic retina and RPE, with prominent underlying choroidal vessels visible in the macula.

One strategy for the treatment of GA is the interruption of the visual cycle by reducing the accumulation of toxic metabolites, A2E and lipofuscin, Acucela launched its Phase 2 clinical trial of emixustat in patients with dry AMD in January 2010. In March 
2010, emixustat was granted Fast Track status by the U.S. FDA for the treatment of dry AMD. In this 90-day, dose-escalation safety study, 72 subjects with GA received either emixustat $(2,5,7$, or $10 \mathrm{mg}$ in the morning or $5 \mathrm{mg}$ in the evening) or placebo once per day. 54 subjects received emixustat and 18 received placebo. Approximately four hours after subjects were given either emixustat or placebo, and following pupil dilation and dark adaptation, ERG measurements were recorded at multiple time points after three minutes of exposure to a bright, bleaching light. Rod photoreceptor b-wave sensitivity was suppressed in a dose-dependent manner in subjects receiving emixustat, as measured by ERG, for doses up to $10 \mathrm{mg}$ (range $26 \%$ - $89 \%$ suppression of b-wave in the $2 \mathrm{mg}$ and $10 \mathrm{mg}$ groups respectively). This effect was reversible, as the post-bleach mean b-wave amplitudes returned to baseline 7-14 days after treatment, with exception of the $10 \mathrm{mg}$ group, whose results were influenced by an outlier. There was no significant effect of emixustat on the cone photoresponse as measured by single flash or 31-Hz flicker ERG. Ocular adverse events were noted in a dose-dependent manner, and included chromatopsia (57\% of emixustat group) and delayed dark-adaptation ( $48 \%$ of emixustat group). $85 \%$ of these adverse events resolved within 7-14 days of discontinuing emixustat [53].

In May 2016, Acucela announced the results of its Phase 2b/3 "S.E.A.T.T.L.E." clinical trial. This study enrolled 508 patients with GA due AMD and sought to demonstrate that emixustat could reduce the growth rate of GA compared to placebo. The lesion growth rates over 24 months for the $10 \mathrm{mg}, 5 \mathrm{mg}, 2.5 \mathrm{mg}$ and placebo groups were $1.84 \mathrm{~mm}^{2} /$ year, $1.83 \mathrm{~mm}^{2} /$ year, $1.69 \mathrm{~mm}^{2} /$ year, and $1.69 \mathrm{~mm}^{2} /$ year, respectively. The study failed to meet its primary endpoint, as there was no statistically significant 
difference in lesion growth rate for any treatment group compared to placebo. There was no significant difference in the mean change of BCVA from baseline to month 24 between treatment groups. There was a small numerical treatment difference observed in certain patients with specific genetic profiles in favor of emixustat. The profile of adverse events was similar to that of earlier trials [54]. Acucela has broadened its scope to testing emixustat as treatment for SMD, and approximately 30 patients are enrolled in a phase $2 \mathrm{a}$ study that will take place in the United States [53].

\subsection{Vitamin A Transport}

\subsubsection{Fenretinide}

Fenretinide (Sirion Therapeutics) is an oral synthetic retinoid derivative that competes with retinol to bind with retinol-binding protein 4 (RBP4), thus preventing transport of retinol into the RPE. Serum retinol is maintained in circulation as a tertiary complex with RBP4 and transthyretin (TTR). Reduction in delivery of retinol-RBP-TTR to the RPE is thought to decrease accumulation of A2E and potentially slow the rate of GA growth. Once fenretinide binds to RBP, the RBP-fenretinide complex is rapidly eliminated in the urine [55]. Fenretinide has been shown to reduce formation of A2E in a mouse model of SMD [56]. Possible downsides to fenretinide therapy include its tendency to induce apoptosis in many cell types (including RPE) [57], along with teratogenic effects that would limits its use in women of child-bearing age (more pertinent in treating SMD than AMD) [58].

A recent phase 2 study assessed fenretidine (100 and $300 \mathrm{mg}$ orally administered daily versus placebo) for slowing lesion growth in 246 patients with GA. There was a 
dose-dependent reduction of serum RBP in fenretinide-treated patients. There was also a trend for reduced annual lesion growth rates in patients in the $300 \mathrm{mg}$ fenretinide group who achieved serum retinol levels of $\leq 1 \mu \mathrm{M}\left(1.70 \mathrm{~mm}^{2} /\right.$ year vs. $2.03 \mathrm{~mm}^{2} /$ year, mean reduction of $0.33 \mathrm{~mm}^{2}$ compared to placebo, $\mathrm{p}=0.1848$ ). Only $51 \%$ of patients receiving $300 \mathrm{mg}$ and completing the 2-year study achieved this level of serum retinol reduction, resulting in a non-significant change in lesion growth rate versus the placebo group. RBP reductions $<2 \mathrm{mg} / \mathrm{dL}$ correlated with further reductions in lesion growth rates $\left(\mathrm{r}^{2}=\right.$ 0.478). There was a $45 \%$ reduction in CNV formation among fenretinide groups, though all groups in the study lost a mean of 10-11 letters of vision at 2 year follow up, consistent with the natural history of GA and suggesting no visual benefit to the modest reduction in GA growth [59]. The $300 \mathrm{mg}$ fenretinide group had $20.2 \%$ of patients withdraw from the study due to adverse effects, though only complaints related to the skin or eye were thought to be drug related. The most common ocular adverse events that were reported (but did not necessarily lead to study withdrawal) included decreased visual acuity (71\%), night blindness (37.3\%), and visual disturbance (26.5\%).

\subsubsection{A1120}

A1120 (ICR-14967) is another drug that aims to lower serum retinol levels to treat AMD and SMD. It was originally developed as a potential treatment for diabetes. Like fenretinide, A1120 is a RBP4 antagonist, though A1120 differs in that it is not a retinoid and not an agonist to Retinoic Acid Receptor-alpha. This property may spare patients from the side effect profile associated with retinoids, which includes nyctalopia and delayed dark adaptation. $\mathrm{ABCA} 4^{-/-}$mice that were administered A1120 $30 \mathrm{mg} / \mathrm{kg}$ 
daily for 6 weeks were found to have a $75 \%$ reduction of serum RBP4 along with $50 \%$ reduction of lipofuscin bisretinoids compared to the control group. On ERG testing, no statistically significant difference in kinetics of the b-wave recovery after photobleaching was found between the groups of A1120- and vehicle-treated wild-type animals, suggesting that A1120's capacity for reduction of lipofuscin bisretinoids in the retina may not be associated with the appreciable suppression of the visual cycle. This finding supports the notion that A1120 is unlikely to cause side effects of nyctalopia or delayed dark-adaptation, which is seen in the other visual cycle modulators [60]. Based on this preclinical data, clinical trials are under development by a collaborative effort between iCura Vision, Columbia University, and the National Institutes of Health [60].

\section{Conclusion}

Pharmacological modulation of the visual cycle serves as a novel approach to the treatment of degenerative retinal diseases. Four classes of therapeutics, which work by inhibiting vitamin A dimer and lipofuscin accumulation in the retina, are emerging as potential treatments for SMD and AMD. These include direct inhibitors of key visual cycle enzymes (isotretinoin and emixustat), RBP4 antagonists (fenretinide and A1120), primary amine-containing aldehyde traps (VM200), and deuterated analogs of vitamin A (ALK-001). For treatment of RP and LCA, 9-cis-retinyl acetate (zuretinol) and alga Dunaliella bardawil aim to bypass inherited defects in RPE65 and LRAT function in order to regenerate the visual chromophore 11-cis-retinal, thus allowing the next photon of light to initiate the next visual cycle. 


\section{Expert Opinion (500-1000 words)}

The pathophysiology of AMD is very complex and not fully elucidated, and would thus require a multi-targeted approach to maximally halt the disease process. Oxidative damage, chronic inflammation, malfunction of the complement system, betaamyloid deposition, and lipofuscin accumulation are all thought to contribute to AMD progression to varying degrees. For this reason, several different classes of medications are being tested to target these pathways, including mammalian target of rapamycin (mTOR) inhibitors, complement inhibitors, matrix metalloproteinase inhibitors, lowdensity lipoprotein (LDL)-lowering drugs, anti-amyloid beta drugs, antioxidants, neuroprotectants, choroidal perfusion enhancers, neurotrophins, and stem cell therapy [61]. Visual cycle modulation is just one piece of this convoluted puzzle, and many questions remain regarding its efficacy in slowing progression of GA. The emixustat and fenretinide trials both failed to show a statistically significant improvement in GA progression or BCVA, while also causing a notable amount of ocular and non-ocular side effects.

For management of both SMD and AMD, A1120 may one day serve as an intriguing option, as its developer suggests that it may not be associated with mechanismbased ocular side effects typical for direct visual cycle inhibitors, such as nyctalopia and delayed dark-adaptation [62]. However, given that it has only been tested in mouse models of SMD, it is too soon to speculate about its efficacy in humans. Aldehyde trapping (VM200) could also represent an appealing approach to inhibiting retinal bisretinoid formation, as it may also lack the mechanism-based ocular side effects typical for direct visual cycle inhibitors. However, the compounds may need to be administered 
at very high systemic doses to act as aldehyde traps in the retina, which raises safety concerns. Local retinal delivery may be considered as an alternative to systemic administration of aldehyde traps to overcome potential systemic toxicities [62].

Isotretinoin, which has been used as an acne treatment for many years with an acceptable safety proposal (aside from teratogenicity), has shown some promise in reducing lipofuscin formation in mouse models of SMD, but there is a paucity of data to confirm its beneficial effect on humans with SMD at this point in time. C20-D3-vitamin A (ALK-001) has shown similarly impressive results in reducing accumulation of Vitamin A dimers and lipofuscin in the retina, along with improved ERG function in Stargardt mouse models, though the results of the completed phase 1 trial have not been released, to the best of the authors' knowledge.

There are many different genetic mutations that may cause RP and LCA, so much like AMD, there is no one-size-fits-all treatment for this group of diseases. Modulation of the visual cycle with retinoids such as 9-cis-retinyl-acetate (zuretinol) and 9-cis- $\beta$ carotene may help those patients with mutations in LRAT or RPE65. Human trials, while limited in sample size, have demonstrated that some RP and LCA patients taking zuretinol have shown improvements in visual field and visual acuity. However, given that over 100 genes account for about $70 \%$ of RP patients while the remainder have unknown mutations [3], it is likely that only a small subset of patients would be candidates for the therapies mentioned in this article. Given the recent success of viral vector gene therapy for LCA, it will be interesting to see if oral retinoids will have a supplementary role in patients with LCA who opt for gene therapy, or if pharmacotherapy will be bypassed altogether. 


\section{Article Highlights Box}

- Degenerative retinal diseases such as dry age-related macular degeneration (AMD), retinitis pigmentosa (RP), Leber's congenital amaurosis (LCA), and Stargardt macular dystrophy (SMD) may cause blindness and currently lack effective therapies.

- Oral retinoid therapies such as 9-cis-retinyl and 9-cis- $\beta$-carotene may help to restore 11-cis-retinal levels in cases of RP and LCA caused by mutations in LRAT and RPE65. Phase 3 clinical trial data will be needed to definitively determine improvements in visual acuity and visual fields.

- Oral therapies for SMD aim to decrease accumulation of Vitamin A dimers and lipofuscin in the retina and RPE, and include ALK-001, isotretinoin, VM200, emixustat, and A1120. There is an abundance of data that shows efficacy of these treatments in mouse models of SMD, though evidence of efficacy in humans is currently lacking.

- Visual cycle suppression is associated with nyctalopia, delayed dark-adaptation, and dyschromatopsia.

- Fenretinide, emixustat and A1120 are visual cycle modulators (VCMs) under investigation for Dry AMD, though none of them has been shown to reduce geographic atrophy or improve vision in humans.

- Gene therapy with viral vectors is being explored as another option in treating RP, LCA, SMD, and AMD.

\section{Bibliography}


Papers of note have been highlighted as either of interest $(*)$ or of considerable interest

$(* *)$ to readers

1. Saari JC. Vitamin A metabolism in rod and cone visual cycles. Annu Rev Nutr 2012; 32:125-145.

** Review of visual cell cycle phsyiology, which is necessary to understand the role of visual cell cycle modulators in treating retinal disease

2. Lorenz B, Gyurus P, Preising M, et al. Early-onset severe rod-cone dystrophy in young children with RPE65 mutations. Invest Ophthalmol Vis Sci 2000; 41:2735-2742.

3. Hartong DT, Berson EL, Dryja TP. Retinitis pigmentosa. Lancet 2006; 368:1795-1809.

4. Hamel C. Retinitis pigmentosa. Orphanet J Rare Dis 2006; 1:40.

5. Stone EM. Leber congenital amaurosis - a model for efficient genetic testing of heterogeneous disorders: LXIV Edward Jackson Memorial Lecture. Am J Ophthalmol 2007; 144:791-811.

6. Gu SM, Thompson DA, Srikumari CR, et al. Mutations in RPE65 cause autosomal recessive childhood-onset severe retinal dystrophy. Nat Genet 1997; 17:194-197.

7. Thompson DA, Li Y, McHenry CL, et al. Mutations in the gene encoding lecithin retinol acyltransferase are associated with early-onset severe retinal dystrophy. Nat Genet 2001; 28:123-124.

8. Redmond TM, Yu S, Lee E, et al. Rpe65 is necessary for production of 11-cisvitamin A in the retinal visual cycle. Nat Genet 1998; 20:344-351.

9. Maeda T, Cideciyan AV, Maeda A, et al. Loss of cone photoreceptors caused by chromophore depletion is partially prevented by the artificial chromophore pro-drug, 9-cis-retinyl acetate. Hum Mol Genet 2009; 18:2277-2287.

10. Van Hooser JP, Aleman TS, He YG, et al. Rapid restoration of visual pigment and function with oral retinoid in a mouse model of childhood blindness. Proc Natl Acad Sci U S A 2000; 97:8623-8628.

11. Maeda T, Dong Z, Jin H, et al. QLT091001, a 9-cis-retinal analog, is welltolerated by retinas of mice with impaired visual cycles. Invest Ophthalmol Vis Sci 2013; 54:455-466.

12. Batten ML, Imanishi Y, Tu DC, et al. Pharmacological and rAAV gene therapy rescue of visual functions in a blind mouse model of Leber congenital amaurosis. PLoS Med 2005; 2:e333.

13. Scholl HP, Moore AT, Koenekoop RK, et al. Safety and Proof-of-Concept Study of Oral QLT091001 in Retinitis Pigmentosa Due to Inherited Deficiencies of Retinal Pigment Epithelial 65 Protein (RPE65) or Lecithin:Retinol Acyltransferase (LRAT). PLoS One 2015; 10:e0143846.

14. Bainbridge JW, Smith AJ, Barker SS, et al. Effect of gene therapy on visual function in Leber's congenital amaurosis. N Engl J Med 2008; 358:2231-2239. 
15. Maguire AM, Simonelli F, Pierce EA, et al. Safety and efficacy of gene transfer for Leber's congenital amaurosis. N Engl J Med 2008; 358:2240-2248.

16. Cideciyan AV, Aleman TS, Boye SL, et al. Human gene therapy for RPE65 isomerase deficiency activates the retinoid cycle of vision but with slow rod kinetics. Proc Natl Acad Sci U S A 2008; 105:15112-15117.

17. Koenekoop RK, Sui R, Sallum J, et al. Oral 9-cis retinoid for childhood blindness due to Leber congenital amaurosis caused by RPE65 or LRAT mutations: an open-label phase $1 \mathrm{~b}$ trial. Lancet 2014; 384:1513-1520.

** Human trial testing the efficacy of oral 9-cis-retinoid, showing that it was generally well tolerated and could improve visual fields and visual acuity in patients with LCA

18. Age-Related Eye Disease Study 2 Research G. Lutein + zeaxanthin and omega3 fatty acids for age-related macular degeneration: the Age-Related Eye Disease Study 2 (AREDS2) randomized clinical trial. JAMA 2013; 309:20052015.

19. Rotenstreich Y, Belkin M, Sadetzki S, et al. Treatment with 9-cis betacarotene-rich powder in patients with retinitis pigmentosa: a randomized crossover trial. JAMA Ophthalmol 2013; 131:985-992.

20. Wang XD, Krinsky NI, Benotti PN, et al. Biosynthesis of 9-cis-retinoic acid from 9-cis-beta-carotene in human intestinal mucosa in vitro. Arch Biochem Biophys 1994; 313:150-155.

21. Paiva SA, Russell RM. Beta-carotene and other carotenoids as antioxidants. J Am Coll Nutr 1999; 18:426-433.

22. Chung DC, McCague S, Yu ZF, et al. Novel mobility test to assess functional vision in patients with inherited retinal dystrophies. Clin Exp Ophthalmol 2017.

23. Russell S, Bennett J, Wellman JA, et al. Efficacy and safety of voretigene neparvovec (AAV2-hRPE65v2) in patients with RPE65-mediated inherited retinal dystrophy: a randomised, controlled, open-label, phase 3 trial. Lancet 2017; 390:849-860.

** Phase 3 trial data that demonstrated RPE65 gene therapy significantly improved performance on the multi-luminance mobility test in patients with Leber's congenital amaurosis

24. Therapeutics S. Spark Therapeutics Completes Rolling Biologics License Application Submission to FDA for Investigational Gene Therapy Voretigene Neparvovec. Available at: http://ir.sparktx.com/phoenix.zhtml?c=253900\&p=irolnewsArticle\&cat=news\&id=2273905 2017.

25. Age-related macular degeneration. Available at: https://http://www.agtc.com/products/macular-degeneration.

26. iCura Vision Licenses First-in-Class Therapeutic Program to Treat Dry Macular Degeneration from Columbia University in Collaboration with National Institutes of Health. In.

27. Rotenstreich Y, Fishman GA, Anderson RJ. Visual acuity loss and clinical observations in a large series of patients with Stargardt disease.

Ophthalmology 2003; 110:1151-1158. 
28. Fishman GA, Farber M, Patel BS, et al. Visual acuity loss in patients with Stargardt's macular dystrophy. Ophthalmology 1987; 94:809-814.

29. Brunk UT, Terman A. Lipofuscin: mechanisms of age-related accumulation and influence on cell function. Free Radic Biol Med 2002; 33:611-619.

30. Gray DA, Woulfe J. Lipofuscin and aging: a matter of toxic waste. Sci Aging Knowledge Environ 2005; 2005:re1.

31. de Jong PT. Age-related macular degeneration. N Engl J Med 2006; 355:14741485.

32. Sparrow JR, Boulton M. RPE lipofuscin and its role in retinal pathobiology. Exp Eye Res 2005; 80:595-606.

33. Weng J, Mata NL, Azarian SM, et al. Insights into the function of Rim protein in photoreceptors and etiology of Stargardt's disease from the phenotype in abcr knockout mice. Cell 1999; 98:13-23.

34. Sparrow JR, Fishkin N, Zhou J, et al. A2E, a byproduct of the visual cycle. Vision Res 2003; 43:2983-2990.

35. Lamb LE, Simon JD. A2E: a component of ocular lipofuscin. Photochem Photobiol 2004; 79:127-136.

36. Suter M, Reme C, Grimm C, et al. Age-related macular degeneration. The lipofusion component N-retinyl-N-retinylidene ethanolamine detaches proapoptotic proteins from mitochondria and induces apoptosis in mammalian retinal pigment epithelial cells. J Biol Chem 2000; 275:3962539630.

37. Finnemann SC, Leung LW, Rodriguez-Boulan E. The lipofuscin component A2E selectively inhibits phagolysosomal degradation of photoreceptor phospholipid by the retinal pigment epithelium. Proc Natl Acad Sci U S A 2002; 99:3842-3847.

38. Kaufman Y, Ma L, Washington I. Deuterium enrichment of vitamin A at the C20 position slows the formation of detrimental vitamin A dimers in wildtype rodents. J Biol Chem 2011; 286:7958-7965.

39. Ma L, Kaufman Y, Zhang J, et al. C20-D3-vitamin A slows lipofuscin accumulation and electrophysiological retinal degeneration in a mouse model of Stargardt disease. J Biol Chem 2011; 286:7966-7974.

40. Charbel Issa P, Barnard AR, Herrmann P, et al. Rescue of the Stargardt phenotype in Abca4 knockout mice through inhibition of vitamin A dimerization. Proc Natl Acad Sci U S A 2015; 112:8415-8420.

41. Phase 1 Safety Study of ALK-001 in Healthy Volunteers. Accessed from : https:/www.clinicaltrials.gov/ct2/show/NCT02230228

42. Phase 2 Tolerability and Effects of ALK-001 on Stargardt Disease (TEASE). Accessed from: https://clinicaltrials.gov/ct2/show/NCT02402660

43. Maeda A, Golczak M, Chen Y, et al. Primary amines protect against retinal degeneration in mouse models of retinopathies. Nat Chem Biol 2011; 8:170178.

44. Vision Medicines Overview of VM200. Accessed from: http://visionmedicines.com/pdf/VM200-Overview-Presentation.pdf

45. Han Z, Conley SM, Naash MI. Gene therapy for Stargardt disease associated with ABCA4 gene. Adv Exp Med Biol 2014; 801:719-724. 
46. Lu LJ, Liu J, Adelman RA. Novel therapeutics for Stargardt disease. Graefes Arch Clin Exp Ophthalmol 2017; 255:1057-1062.

47. Moore NA, Morral N, Ciulla TA, et al. Gene therapy for inherited retinal and optic nerve degenerations. Expert Opin Biol Ther 2017:1-13.

48. Radu RA, Mata NL, Nusinowitz S, et al. Treatment with isotretinoin inhibits lipofuscin accumulation in a mouse model of recessive Stargardt's macular degeneration. Proc Natl Acad Sci U S A 2003; 100:4742-4747.

49. Weleber RG, Denman ST, Hanifin JM, et al. Abnormal retinal function associated with isotretinoin therapy for acne. Arch Ophthalmol 1986; 104:831-837.

50. Sieving PA, Chaudhry P, Kondo M, et al. Inhibition of the visual cycle in vivo by 13-cis retinoic acid protects from light damage and provides a mechanism for night blindness in isotretinoin therapy. Proc Natl Acad Sci U S A 2001; 98:1835-1840.

51. Kubota R, Boman NL, David R, et al. Safety and effect on rod function of ACU4429 , a novel small-molecule visual cycle modulator. Retina 2012; 32:183188.

52. Friedman DS, O'Colmain BJ, Munoz B, et al. Prevalence of age-related macular degeneration in the United States. Arch Ophthalmol 2004; 122:564-572.

53. Dugel PU, Novack RL, Csaky KG, et al. Phase ii, randomized, placebocontrolled, 90-day study of emixustat hydrochloride in geographic atrophy associated with dry age-related macular degeneration. Retina 2015; 35:1173-1183.

* Phase $2 b / 3$ trial data that demonstrated lack of efficacy of emixustat in slowing geographic atrophy progression in patients with AMD

54. Acucela Announces Top-Line Results from Phase 2b/3 Clinical Trial of Emixustat. Accessed from: http://www.businesswire.com/news/home/20160525006550/en/AcucelaAnnounces-Top-Line-Results-Phase-2b3-Clinical

55. Berni R, Formelli F. In vitro interaction of fenretinide with plasma retinolbinding protein and its functional consequences. FEBS Lett 1992; 308:43-45.

56. Radu RA, Han Y, Bui TV, et al. Reductions in serum vitamin A arrest accumulation of toxic retinal fluorophores: a potential therapy for treatment of lipofuscin-based retinal diseases. Invest Ophthalmol Vis Sci 2005; 46:4393-4401.

57. Samuel W, Kutty RK, Nagineni S, et al. N-(4-hydroxyphenyl)retinamide induces apoptosis in human retinal pigment epithelial cells: retinoic acid receptors regulate apoptosis, reactive oxygen species generation, and the expression of heme oxygenase-1 and Gadd153. J Cell Physiol 2006; 209:854865.

58. Turton JA, Willars GB, Haselden JN, et al. Comparative teratogenicity of nine retinoids in the rat. Int J Exp Pathol 1992; 73:551-563.

* $\quad$ Phase 2 study that demonstrated lack of efficacy of oral fenretinitde in slowing geographic atrophy progression in patietns with AMD. 
59. Mata NL, Lichter JB, Vogel R, et al. Investigation of oral fenretinide for treatment of geographic atrophy in age-related macular degeneration. Retina 2013; 33:498-507.

60. Dobri N, Qin Q, Kong J, et al. A1120, a nonretinoid RBP4 antagonist, inhibits formation of cytotoxic bisretinoids in the animal model of enhanced retinal lipofuscinogenesis. Invest Ophthalmol Vis Sci 2013; 54:85-95.

61. Holz FG, Strauss EC, Schmitz-Valckenberg S, et al. Geographic atrophy: clinical features and potential therapeutic approaches. Ophthalmology 2014; 121:1079-1091.

62. Petrukhin K. Pharmacological inhibition of lipofuscin accumulation in the retina as a therapeutic strategy for dry AMD treatment. Drug Discov Today Ther Strateg 2013; 10:e11-e20.

\section{Figure Legends:}

Figure 1. The visual cycle pathway begins when light (starburst shape) interacts with rhodopsin (diamond shape), setting off a series of steps catalyzed by enzymes (cloud shapes). The chemical byproducts and investigational drugs are represented in box and oval shapes, respectively. The cross-through symbol denotes an inhibitory effect of a drug on the enzymes, while a plus sign indicates the effect of increasing rhodopsin levels. $\mathrm{RDH}=$ retinol dehydrogenase, $\mathrm{ABCA} 4=\mathrm{ATP}-\mathrm{Binding}$ Cassette Subfamily A Member 4, LRAT $=$ lecithin retinol acyltransferase, $\mathrm{RPE}=$ retinal pigment epithelium.

Figure 2. Montage fundus photo of a patient with retinitis pigmentosa, which demonstrates the classic triad of optic disc pallor, retinal vessel attenuation, and "bonespicule" pigmentary changes in the retinal periphery

Figure 3. Montage fundus photo of a patient with Leber's congenital amaurosis, which demonstrates retinal vessel attenuation and pigmentary changes similar to those seen in RP. 
Figure 4. Fundus photography of a patient with Stargardt macular dystrophy shows yellowish flecks (representing lipofuscin accumulation in the RPE) that extend outward from the macula.

Figure 5. Fundus photography of a patient with age-related macular degeneration with geographic atrophy, which highlights the atrophic appearance of the retina and RPE in the macula, leading to increased prominence of the underlying choroidal vasculature.

Table 1. Summary of the investigational drugs in development to treat inherited and agerelated degenerative diseases of the retina. 


\begin{tabular}{|c|c|c|c|c|c|}
\hline Medication & Chemical Structure & Mechanism of action & Indication & Company & Stage of development \\
\hline $\begin{array}{l}\text { QLT091001 } \\
\text { (Zuretinol) }\end{array}$ & 9-cis-retinyl-acetate & $\begin{array}{l}\text { Combines with opsin to form } \\
\text { isorhodopsin, thus bypassing defects in } \\
\text { the visual cycle }\end{array}$ & $\mathrm{RP} / \mathrm{LCA}$ & $\begin{array}{l}\text { Novelion } \\
\text { Therapeutics }\end{array}$ & Phase 3 trials \\
\hline $\begin{array}{l}\text { Alga Dunaliella } \\
\text { bardawil }\end{array}$ & 9-cis $\beta$-carotene & $\begin{array}{l}\text { 9-cis } \beta \text {-carotene is converted to 9-cis- } \\
\text { retinal in the retina, which then } \\
\text { combines with opsin to form } \\
\text { isorhodopsin }\end{array}$ & $\mathrm{RP}$ & $\begin{array}{l}\text { Available as generic } \\
\text { OTC supplement }\end{array}$ & Approved by US FDA \\
\hline ALK-001 & C20-D3 -vitamin A & $\begin{array}{l}\text { Replacing the C20 hydrogen atoms of } \\
\text { vitamin A with deuterium atoms makes } \\
\text { cleavage of the carbon-hydrogen bond } \\
\text { more difficult, thereby reducing Vitamin } \\
\text { A dimerization and lipofuscin }\end{array}$ & $\begin{array}{l}\text { Stargardt } \\
\text { disease }\end{array}$ & $\begin{array}{l}\text { Alkeus } \\
\text { Pharmaceuticals }\end{array}$ & Phase 2 trials \\
\hline Isotretinoin & 13-cis-retinoic acid & $\begin{array}{l}\text { Inhibits } 11 \text {-cis-retinol dehydrogenase, } \\
\text { thus slowing the synthesis of } 11 \text {-cis- } \\
\text { retinaldehyde and regeneration of } \\
\text { rhodopsin. }\end{array}$ & $\begin{array}{l}\text { Stargardt } \\
\text { disease }\end{array}$ & $\begin{array}{l}\text { available in generic } \\
\text { form }\end{array}$ & Pre-clinical trials \\
\hline VM200 & $\begin{array}{l}\text { enantiomer of } \\
\text { pregabalin }\end{array}$ & $\begin{array}{l}\text { Aldehyde trap (reacts with toxic all-tran- } \\
\text { retinal to form inactive schiff base) }\end{array}$ & $\begin{array}{l}\text { Stargardt } \\
\text { disease }\end{array}$ & Vision Medicine & Pre-clinical trials \\
\hline $\begin{array}{l}\text { ACU-4429 } \\
\text { (Emixustat) }\end{array}$ & $\begin{array}{l}\text { non-retinoid derivative } \\
\text { of retinylamine }\end{array}$ & $\begin{array}{l}\text { Inhibits RPE65, thus reducing the } \\
\text { conversion of all-trans-retinyl ester to } \\
\text { 11-cis-retinol and preventing } \\
\text { accumulation of A2E. }\end{array}$ & $\begin{array}{l}\text { AMD/Stargardt } \\
\text { disease }\end{array}$ & Acucela Inc & $\begin{array}{l}\text { AMD: Phase } 2 \mathrm{~b} / 3 \text { trial } \\
\text { did not meet endpoint. } \\
\text { Stargardt disease: Phase } \\
\text { 2a trial enrolling }\end{array}$ \\
\hline Fenretinide & $\begin{array}{l}\text { synthetic retinoid } \\
\text { derivative, "N-(4- } \\
\text { hydroxyphenyl)retinam } \\
\text { ide" }\end{array}$ & $\begin{array}{l}\text { RBP4 antagonist that prevents transport } \\
\text { of retinol into the RPE }\end{array}$ & AMD & Sirion Therapeutics & $\begin{array}{l}\text { Phase } 2 \text { trial did not } \\
\text { meet primary endpoint }\end{array}$ \\
\hline $\begin{array}{l}\text { A1120 (ICR- } \\
14967)\end{array}$ & $\begin{array}{l}\text { 2-(4-[2- } \\
\text { (trifluoromethyl)phenyl } \\
\text { ]piperidine-1- } \\
\text { carboxamido)benzoic } \\
\text { acid }\end{array}$ & $\begin{array}{l}\text { Nonretinoid RBP4 antagonist that } \\
\text { prevents transport of retinol into the RPE }\end{array}$ & $\begin{array}{l}\text { AMD/Stargardt } \\
\text { disease }\end{array}$ & iCura Vision & $\begin{array}{l}\text { Clinical trials in } \\
\text { development }\end{array}$ \\
\hline
\end{tabular}




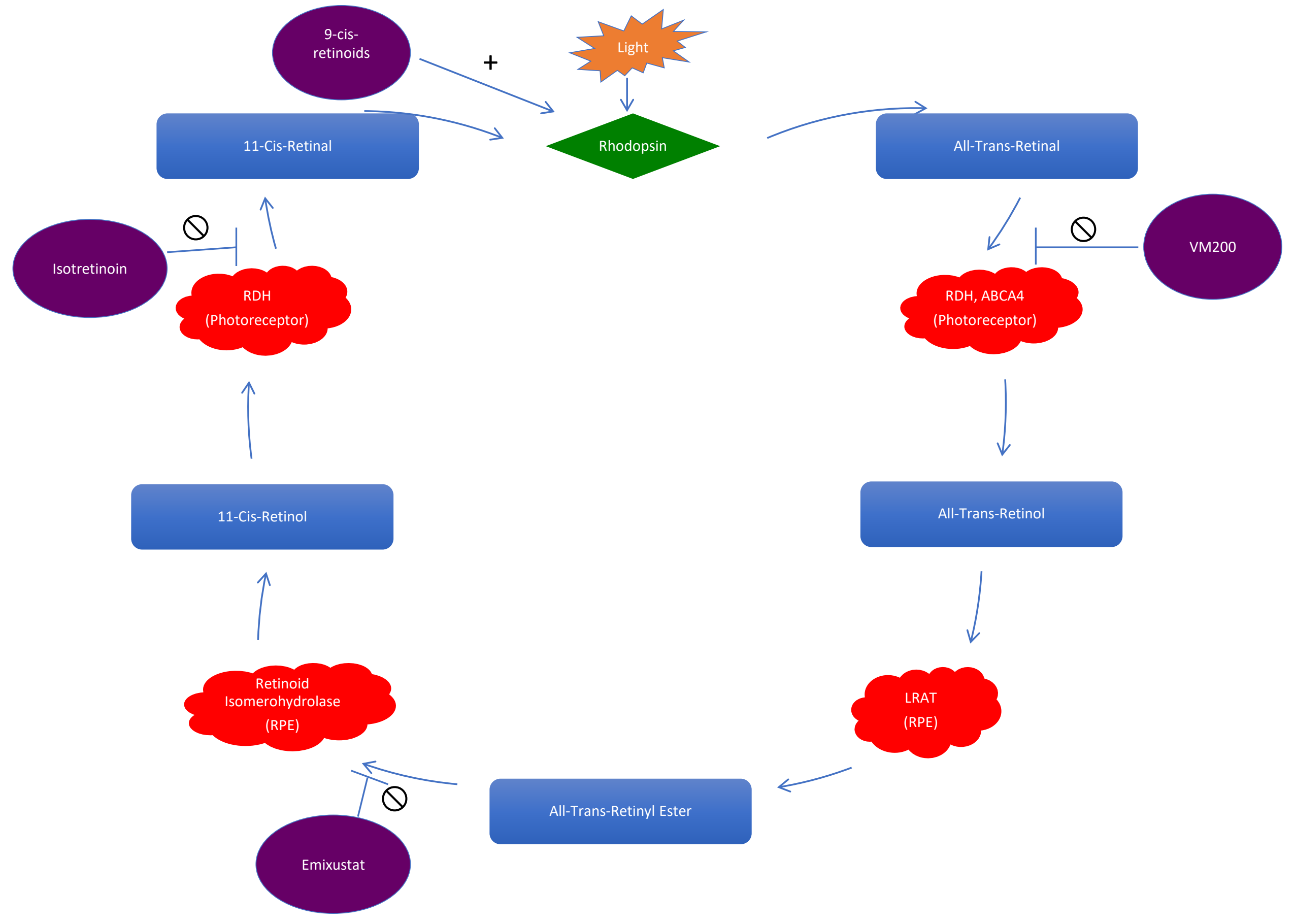

